\title{
Extensive remodeling of the Pseudomonas syringae pv. avellanae type III secretome associated with two independent host shifts onto hazelnut
}

\author{
Heath E O'Brien ${ }^{1 *}$, Shalabh Thakur', Yunchen Gong², Pauline Fung ${ }^{2}$, Jianfeng Zhang ${ }^{2}$, Lijie Yuan²,
} Pauline W Wang ${ }^{1,2}$, Choseung Yong ${ }^{1}$, Marco Scortichini ${ }^{3}$ and David S Guttman ${ }^{1,2}$

\begin{abstract}
Background: Hazelnut (Corylus avellana) decline disease in Greece and Italy is caused by the convergent evolution of two distantly related lineages of Pseudomonas syringae pv. avellanae (Pav). We sequenced the genomes of three Pav isolates to determine if their convergent virulence phenotype had a common genetic basis due to either genetic exchange between lineages or parallel evolution.

Results: We found little evidence for horizontal transfer (recombination) of genes between Pav lineages, but two large genomic islands (GIs) have been recently acquired by one of the lineages. Evolutionary analyses of the genes encoding type III secreted effectors (T3SEs) that are translocated into host cells and are important for both suppressing and eliciting defense responses show that the two Pav lineages have dramatically different T3SE profiles, with only two shared putatively functional T3SEs. One Pav lineage has undergone unprecedented secretome remodeling, including the acquisition of eleven new T3SEs and the loss or pseudogenization of 15, including five of the six core T3SE families that are present in the other Pav lineage. Molecular dating indicates that divergence within both of the Pav lineages predates their observation in the field. This suggest that both Pav lineages have been cryptically infecting hazelnut trees or wild relatives for many years, and that the emergence of hazelnut decline in the 1970s may have been due to changes in agricultural practice.

Conclusions: These data show that divergent lineages of $P$. syringae can converge on identical disease etiology on the same host plant using different virulence mechanisms and that dramatic shifts in the arsenal of T3SEs can accompany disease emergence.
\end{abstract}

Keywords: Effector, Host specificity, Molecular dating

\section{Background}

Pseudomonas syringae is a Gram-negative plant pathogen that causes a spectrum of speck, spot and canker diseases on a range of plant hosts. It is divided into approximately 50 pathovars (pathogenic varieties) that are specialized for particular host plants and are generally unable to cause disease on other species. Multilocus sequence analysis (MLSA) has shown that many

\footnotetext{
*Correspondence: heath.obrien@gmail.com

1 Department of Cell and Systems Biology, University of Toronto, 25 Willcocks St., Toronto, ON M5S 3B2, Canada

Full list of author information is available at the end of the article
}

pathovars correspond to distinct evolutionary (monophyletic) lineages [1,2]. A notable exception to this pattern is $P$. syringae pv. avellanae (Pav), where two distantly related lineages within $P$. syringae have converged upon a common disease phenotype on hazelnut (Corylus avellana) plantations in Greece and Italy. Pav-associated hazelnut decline characterized by wilting of branches and trunk cankers was first observed in Greece and Italy in the mid 1970s, though the disease was not formally described in Italy until the 1990s [3]. MLSA has shown that all isolates from Greece form a distinct lineage related to pathogens of

\section{() Biomed Central}


kiwifruit (P. syringae pv. actinidiae; Pan [4], a.k.a. Psa [5]) and plum (P. syringae pv. morsprunorum; Pmp) in phylogroup 1. This phylogroup also includes a large number of pathogens of herbaceous plants, including the well-studied $P$. syringae pv. tomato strain Pto DC3000. In contrast, Italian isolates collected during outbreaks in the 1990s cluster together in phylogroup 2 , along with pathogens of peas, cereals, and other plants, including the well-studied $P$. syringae pv. syringae strain Psy B728a. More recent outbreaks of hazelnut decline in Italy from 2002-2004 were caused by Pav that phylogenetically clusters with the Greek isolates in phylogroup 1.

In order to determine the genetic changes accompanying the evolution of hazelnut pathogenesis in these two independent lineages, we obtained draft whole genome sequences for the earliest isolate of the hazelnut decline pathogen, Pav BP631, a phylogroup 1 strain isolated from Drama, Greece in 1976 and for Pav Ve013 and Pav Ve037, two strains isolated in Rome, Italy in the early 1990s. The latter two strains represent the extremes of genetic diversity observed in phylogroup $2 \mathrm{Pav}$ strains as determined by the MLSA analysis of Wang et al. [6]. This MLSA analysis indicates that Pav Ve037 clusters with pea pathogens (P. syringae pv. pisi; Ppi) while the other strains group with pathogens of beets ( $P$. syringae pv. aptata; Ptt) and barley (P. syringae pv. japonica; Pja) although with very weak phylogenetic support.

We compared these three draft genome sequences to 27 other complete or draft $P$. syringae genome sequences representing 16 pathovars, including seven phylogroup 1 strains and six phylogroup 2 strains [4,7-17]. We performed ortholog analysis to identify instances of horizontal gene transfer between the two independent Pav lineages and looked in detail at the evolutionary histories of a number of candidate pathogenicity genes, including the type III secreted effectors (T3SEs) that are translocated into host cells and are important for both suppressing and eliciting defense responses. We show that the two lineages have dramatically different T3SE profiles and that Pav BP631 has undergone extensive secretome remodeling.

\section{Results}

\section{Genome sequencing and assembly}

43 million read pairs were generated from the Pav BP631 paired-end library, while the Pav Ve013 and Pav Ve037 paired-end libraries produced 59 million and 35 million read pairs respectively (Table 1 ). The 82 bp reads for the latter two strains resulted in considerably longer contigs (N50s of $31 \mathrm{~kb}$ and $61 \mathrm{~kb}$ ) than the $38 \mathrm{bp} P a v$ BP631 reads (N50 of $6.4 \mathrm{~kb})$. The read depth of the contigs was very uniform for Pav Ve013 and Pav Ve037, with almost all the contigs centered around a depth of 1000X (Figure 1). In contrast, the majority of the Pav BP631 contigs were centered around a depth of 300x, but there were also a large number with depth in the thousands, including some up to almost $10,000 \mathrm{bp}$ in length. These high-coverage contigs indicate that this strain harbors one or more multi-copy plasmids.

When the contigs were scaffolded using 38-45 million mate-pairs, the N50 improved to $79 \mathrm{~kb}$ for Pav BP631 and to 264-298 $\mathrm{kb}$ for the other strains (Table 1). The total genome sizes were 6.6 megabases $(\mathrm{Mb})$ for $\mathrm{Pav}$ BP631 and 6.1 to $6.2 \mathrm{Mb}$ for the other two strains, consistent with the presence of extra-chromosomal plasmids in Pav BP631. Pav Ve013 and Pav Ve037 are largely colinear with the phylogroup 2 reference strain Psy B728a, while Pav BP631 displays substantially more rearrangement relative to Pto DC3000, the reference strain for phylogroup 1 (Figure 2). There is a $95 \mathrm{~kb}$ scaffold in Pav BP631 that is made up of high-coverage contigs and is colinear with plasmid A from Pto DC3000 over about half of its length.

\section{Ortholog analysis}

The RAST annotation sever predicted between 4816 and 5136 open reading frames (ORFs) per strain (Table 1) which were grouped into between 4710 and 4951 ortholog groups by orthoMCL (Figure 3a). There were 3967 ortholog families shared among the three Pav strains, all of which were also found in other strains. Of these, 1856 were found in all 29 P. syringae strains, comprising the operational $P$. syringae core genome. Each Pav strain had between 26 and 115 unique genes that lack orthologs in any

Table 1 Genome statistics for strains sequenced in this study

\begin{tabular}{|c|c|c|c|c|c|c|c|}
\hline Strain & Cluster \# ${ }^{1}$ & Contig \# & Contig N50 & Scaffold \# & Scaffold N50 & Genome size & ORFs \\
\hline \multirow[t]{2}{*}{ PavBP631 } & $43 \mathrm{M}^{2} 38 \mathrm{bp} P \mathrm{PE}$ & 1,613 & 6,420 & 297 & 79,231 & $6,628,588$ & 4816 \\
\hline & $38 \mathrm{M} 38$ bp MP & & & & & & \\
\hline \multirow[t]{2}{*}{ ParVe013 } & 59 M 82 bp PE & 389 & 30,917 & 66 & 297,710 & $6,165,792$ & 5136 \\
\hline & $43 \mathrm{M} 40$ bp MP & & & & & & \\
\hline \multirow[t]{2}{*}{ ParVe037 } & 35 M 82 bp PE & 220 & 61,365 & 61 & 263,756 & $6,050,967$ & 5078 \\
\hline & $45 \mathrm{M} 40 \mathrm{bp} \mathrm{MP}$ & & & & & & \\
\hline
\end{tabular}

1. PE: paired-end (ca. $200 \mathrm{bp}$ insert). MP: mate-pair (3-5 kb insert).

2. Millions of reads. 

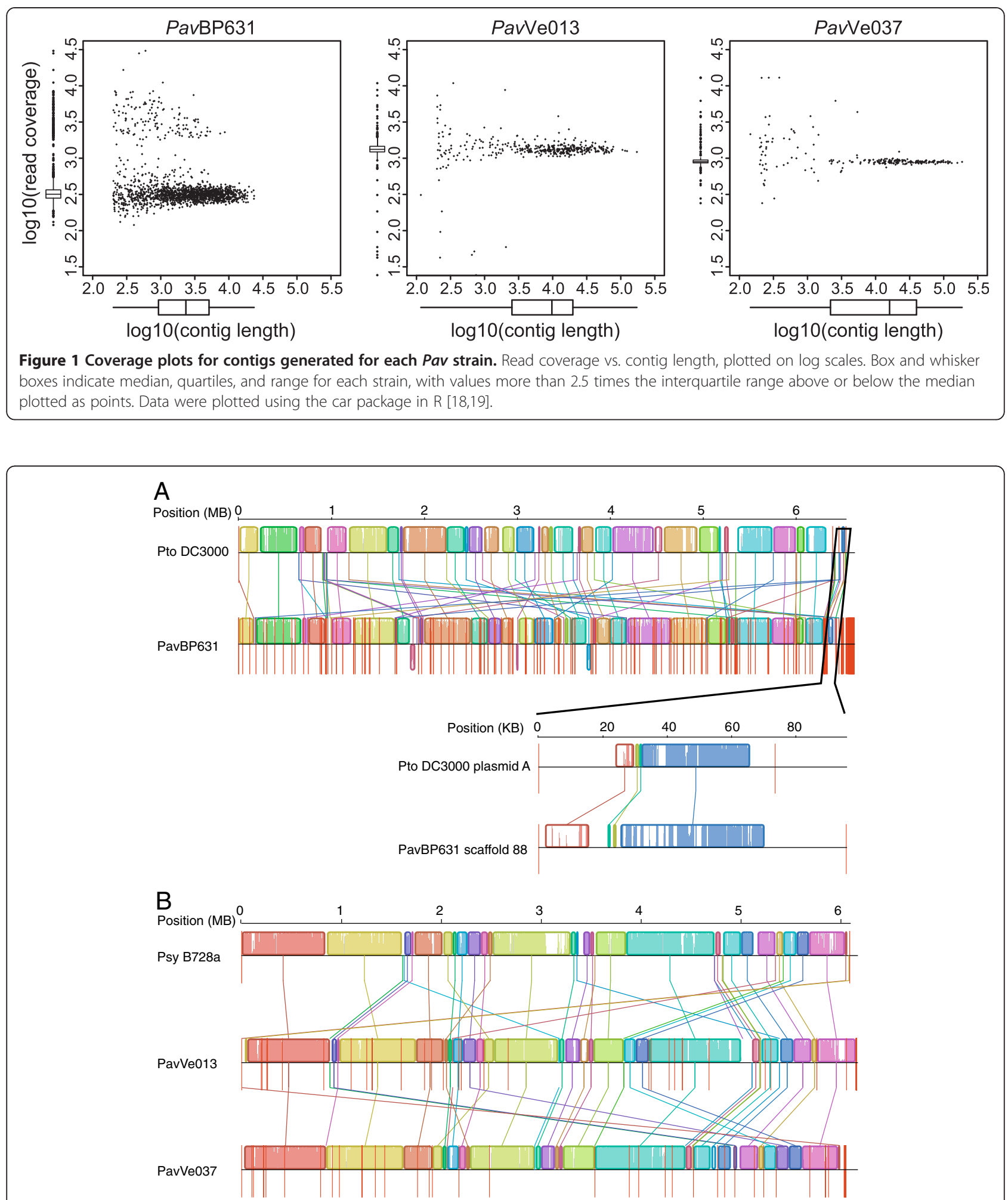

Figure 2 Whole-genome alignments of Pav scaffolds to the most closely related reference sequences. A. PavBP631 contigs aligned to Pto DC3000 reference sequence. Inset: Alignment of scaffold 88 to plasmid A from Pto DC3000 (this was done as a separate analysis). B. Pav Ve013 and Pav Ve037 contigs aligned to Psy B728a reference sequence. Each colored block represents a local colinearity block that can be aligned between strains without any rearrangements. White spaces within blocks indicate regions of low sequence conservation. Vertical red lines indicate scaffold breaks for Pav sequences or boundaries between chromosomes/plasmids in the case of the Pto DC3000 reference sequence. Alignments were generated using progressiveMauve [20]. 


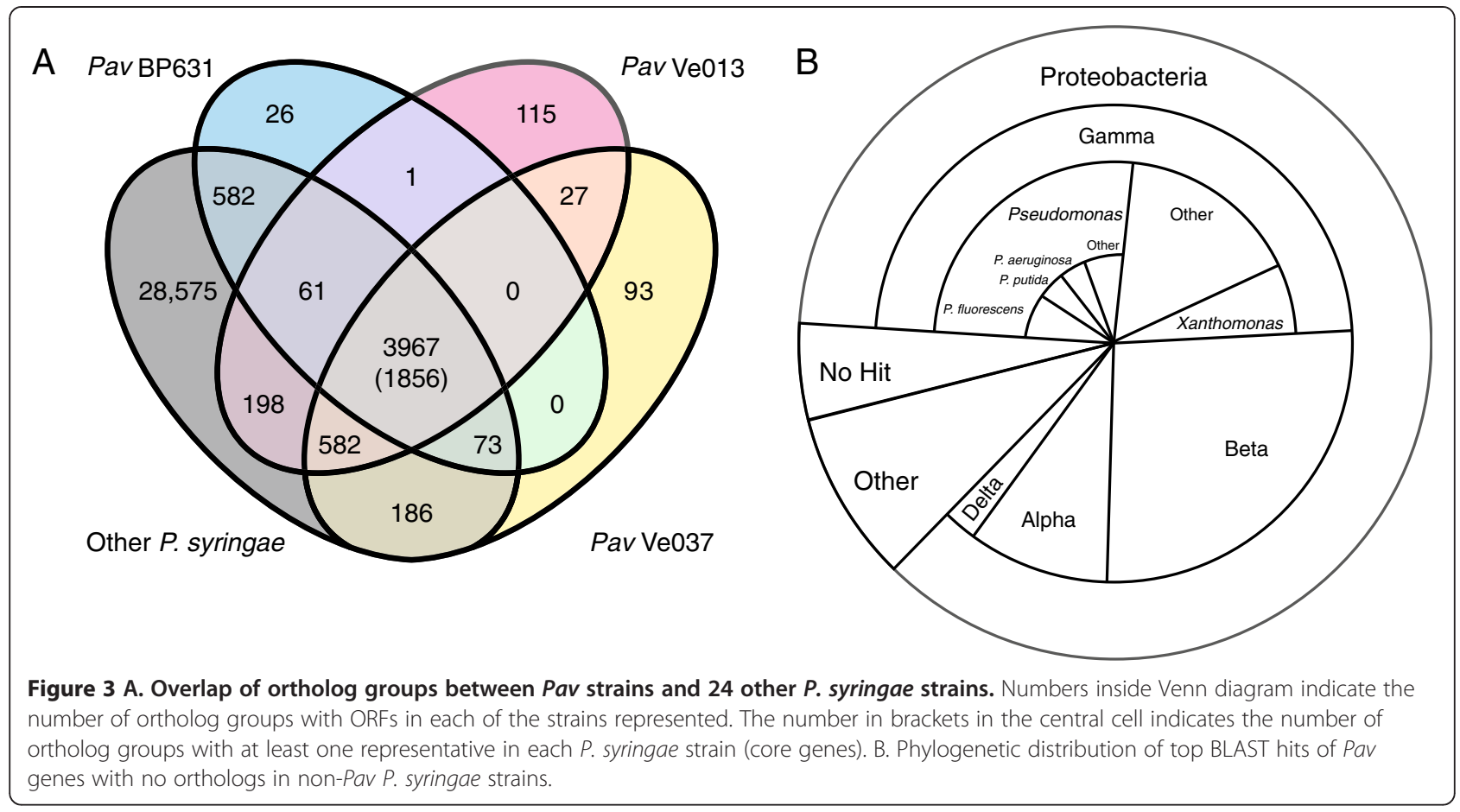

other P. syringae strain. The closely related Pav Ve013 and Pav Ve037 strains shared 27 ORFs that lacked ortho$\operatorname{logs}$ in any other $P$. syringae strain, while there were no ORFs found only in the three Pav strains and no other P. syringae strain.

There were a total of 262 Pav- specific homology groups that lacked orthologs in any other Psy strain in the ortholog analysis section of the results. Approximately half of these were most similar to genes from other species in the gamma-Proteobacteria, while another $25 \%$ were most similar to genes from betaProteobacterial species (Figure 3b). Over half of the ORFs with gamma-Proteobacterial hits matched genes from other Pseudomonas species, while $\sim 15 \%$ were to genes from the plant pathogen Xanthomonas campestris. Of the 142 Pav-specific genes in Pav Ve013, 101 were located in two large gene clusters. One of these was a $110 \mathrm{~kb}$ cluster of 43 genes inserted at a tRNA locus in a region that is syntenic between Pav Ve013 and Psy B728a (Additional file 1: Figure S1). Of these genes, 32 are most similar to Xanthomonas campestris 8004 genes ( $>50 \%$ overlap; E-value $<10^{-10}$ ), including a type IV secretion gene and a transposase gene located at one end of the cluster. The second cluster is $175 \mathrm{~kb}$ in length and consists of 58 genes, including 17 that are shared with Pav Ve037 (Additional file 2: Figure S2). The central core of this region comprises a $49 \mathrm{~kb}$ PFGI-1 type integrative conjugative element (ICE), most of which is homologous to an ICE from Pseudomonas fluorescens SWB25.

\section{Recombination and phylogenetic analysis}

Comparisons of genealogies for each gene greater than $300 \mathrm{bp}$ in length to the genome tree identified seven putatively recombinant genes where Pav BP631 is sister to one or both of the other Pav strains. However, in two cases all but one of the sequences are from Pav strains, so Pav BP631 necessarily has to be sister to other Pav strains in the unrooted tree. Three of the remaining five have very poor branch support. The remaining two putatively recombinant genes, a GAD-like protein and a putative prophage lysozyme, cluster Pav BP631 with one of the other Pav strains, but not both. In both cases the gene trees are highly incongruent with the core genome phylogeny, so it is not possible to determine the direction of transfer. Indeed, there are relatively long terminal branches leading to the $P a v$ strains, suggesting that both $P a v$ strains horizontally acquired the gene from other un-sequenced strains or that their relation may be an artifact of long-branch attraction.

When 42,569 variable positions from 595 single-copy orthologous genes in each of the 29 genome sequences were used for phylogenetic analysis the relationships were consistent with previous MLSA studies, although with much stronger phylogenetic support (Figure 4). There was 100\% approximate Likelihood Ratio Test (aLRT) support for every node except for two of the relationships within the Pto lineage. In phylogroup 1, Pav BP631 clustered with Pan 302091 and Pmo 301020, sister to five Pto strains and Pla 302278. In phylogroup 2, Pav Ve013 and Pav Ve037 cluster as a sister lineage 


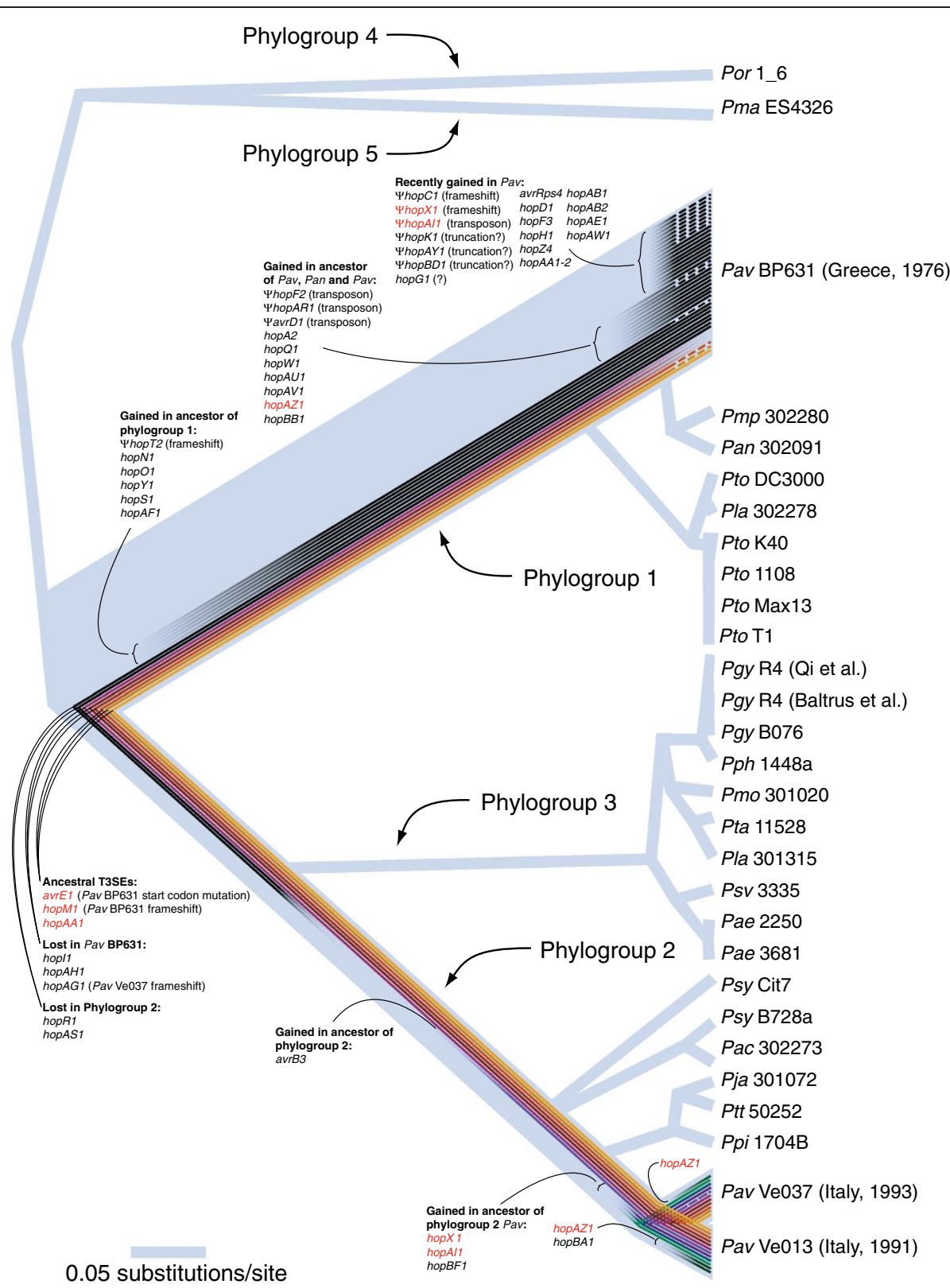

Figure 4 Whole-genome phylogenetic relationships among $P$. syringae strains with evolutionary histories of Pav T3SEs mapped onto branches. Each line within the branches represents one T3SE and indicates when it was acquired or lost by the ancestors of the Pav strains. Dashed lines indicate that a T3SE has become a pseudogene. T3SEs that are present in all Pav strains are indicated in red. Lines representing T3SEs in phylogroup 2 are arbitrarily colored to aid in following them between strains. Phylogroup designations follow [1]. All branches have 100\% aLRT support except for the relationships among Pto strains K40, 1108, Max13 and T1.

to Pja, 301072, Ptt 50252 and Ppi 1704B within a group that also included Psy Cit7, Pac 302273 and Psy B728a. These two phylogroups clustered with the phylogroup 3 lineage that included 10 of the twelve additional sequenced strains, to the exclusion of the single representatives of phylogroups 4 and 5 . The rooting of the tree is uncertain since the phylogenetic analysis did not include outgroups.

\section{Divergence times}

Divergence time estimates were strongly dependent on the substitution rate priors specified (Table 2). Using the slower rate based on the divergence of E. coli from Salmonella 140 million years ago, we obtained age estimates for the most recent common ancestor of all $P$. syringae isolates ranging from 150 to 183 million years, depending on the locus. Phylogroup 1 Pav strains are 
Table 2 Divergence time estimates for Pav lineages

\begin{tabular}{|c|c|c|c|c|c|}
\hline \multirow[t]{2}{*}{ Calibration point } & \multirow{2}{*}{$\begin{array}{l}\text { Rate } \\
\text { (subst./yr) }\end{array}$} & \multirow[t]{2}{*}{ Locus } & \multicolumn{3}{|c|}{ Age of Most Recent Common Ancestor (mean, $95 \% \mathrm{Cl})^{1}$} \\
\hline & & & P. syringae & Phylogroup 1 Pav & Phylogroup 2 Pav \\
\hline \multirow[t]{4}{*}{ E. coli-Salmonella (140 MYA²) [22] } & $1 \times 10^{-9}$ & gapA & 183 MYA (92.8-300) & 3.16 MYA (0.831-6.39) & 29.5 MYA (16.9-44.5) \\
\hline & & $g / t A$ & 171MYA (75.4-300) & 3.88 MYA (0.945-8.02) & 17.6 MYA (7.10-29.8) \\
\hline & & gyrB & 171 MYA (93.7-272) & 10.1 MYA (2.62-19.5) & 34.3 MYA (17.9-54.8) \\
\hline & & $r p o D$ & 153 MYA (66.4-260) & 5.23 MYA (1.61-9.80) & $14.8(7.17-23.1)$ \\
\hline \multirow[t]{4}{*}{ MRSA (1990) [21] } & $2 \times 10^{-6}$ & gapA & $74,000(39,800-116,000)$ & $1200(281-2350)$ & $12,000(7270-17,400)$ \\
\hline & & $g / t A$ & $41,600(22,200-67,400)$ & $1380(414-2690)$ & $4560(2210-7070)$ \\
\hline & & gyr $B$ & $51,900(30,500-77,700)$ & $3400(1050-6480)$ & $10,600(5580-16,700)$ \\
\hline & & $r p o D$ & $49,600(24,400-82,300)$ & $1740(640-3170)$ & $7270(3810-11,700)$ \\
\hline
\end{tabular}

1. Years before present unless otherwise indicated.

2. Million years before present.

inferred to have diverged between 3 and 10 million years ago, while phylogroup 2 strains have ages ranging from 17 to 34 million. When the substitution rate is inferred from the emergence of a clonal lineage of methicillinresistant Staphylococcus aureus (MRSA) since 1990 [21], $P$. syringae is inferred to have diversified within the last 42,000 to 74,000 years. Even with this rapid rate the data are not consistent with emergence of Pav within the last 40 years as the minimum age within the $95 \%$ confidence interval of any of the loci is 281 years for phylogroup 1 Pav and 2210 years for phylogroup 2 Pav. Phylogroup 2 $P a v$ is inferred to have emerged thousands of years before phylogroup 1 Pav (4500-12,000 years versus 12001700 years).

\section{Type III secreted effectors}

There are dramatic differences in the number of T3SE homologs encoded in the genome of Pav BP631 versus the two other strains (Figure 4). Pav BP631 has homologs of 38 T3SEs, of which five have frameshift mutations and four have transposon insertions. There are partial sequences of three additional T3SEs, suggesting that they are truncated. However, they are located at the ends of scaffolds, so we are unable to confirm this. The entire sequence of a fourth T3SE that is also located at the end of a scaffold, hop G1, is present except for the stop codon. In contrast, Pav Ve013 and Pav Ve037 have homologs of only twelve and eleven T3SEs respectively, and one of these, hopAG1, is disrupted by a frameshift in Pav Ve037.

Only six T3SE homologs are common to all three Pav strains, and four of these are putatively non-functional in Pav BP631. Three of these shared T3SEs (avrE1, hopM1, and hopAA1) are also present in all other P. syringae strains and have genealogical histories congruent with the core genome phylogeny of the species, though hopM1 is truncated in many strains. These three T3SEs are located in the conserved effector locus (CEL) that flanks the type III secretion system structural genes. The
Pav BP631 hopM1 locus has a number of frameshift mutations, while the avrE1 gene contains a mutation in the first codon, changing GTG to GTA, which is a highly-atypical start codon that very likely severely reduces or completely disrupts translation [23]. The only shared and putatively functional T3SE in the CEL is hopAA1.

The other T3SE homologs that are present in all three $P a v$ strains are hopAI1, which is truncated in $P a v$ BP631, hopX1, which has a frameshift in Pav BP631, and hopAZ1. All three Pav strains carry hopX1 in the exchangeable effector locus (EEL), which is located on the opposite side of the type III secretion system structural genes as the CEL, and which contains a variable assortment of T3SEs that are flanked by conserved genes. The EEL of Pav Ve013 and Pav Ve037 also contain avrB3 while the EEL of Pav BP631 contains a hopF2 sequence that has been disrupted by a transposase. Both hopX1 and hopAI1 appear to have been acquired independently by the two Pav lineages after their divergence from their most recent non-Pav common ancestor. The hopAZ1 T3SE is particularly interesting since it is intact and putatively functional in all three Pav strains, yet appears to have been acquired independently by all three. No Pav HopAZ1 sequence shares more than $71 \%$ amino acid identity with any other Pav sequence, and they each form very strongly supported distinct phylogenetic clusters with other HopAZ1 alleles (Additional file 3: Figure S3).

Five other T3SEs are present in the majority of $P$. syringae strains and have phylogenies congruent with the core genome. These include two that were lost in the common ancestor of all phylogroup 2 strains (hopR1 and hopAS1) and three that have recently been lost in the phylogroup 1 Pav lineage (hopI1, hopAH1 and hopAG1). All other Pav T3SEs have been acquired by horizontal transfer since the two Pav lineages diverged from each other. In the phylogroup 2 lineage, avrB3 was acquired by the common ancestor of all phylogroup 2 
strains, hopBF1 was acquired by the common ancestor of phylogroup $2 \mathrm{Pav}$, and hopBA1 was acquired by $P a v$ $\mathrm{Ve} 013$ since its divergence from $\mathrm{Pav}$ Ve037. In the phylogroup 1 lineage, six T3SEs were acquired by the common ancestor of all phylogroup 1 strains. Nine additional T3SEs (plus hopAZ1) were acquired by the common ancestor of Pav BP631, Pmp 302280 and Pan 302191. However, the majority of T3SE gain has occurred since Pav BP631 diverged from its common ancestor with Pmp 302280 and Pan 302191 (15, plus hopX1 and hopAI1), almost half of which are pseudogenes.

\section{Discussion}

The hazelnut decline pathogen $P$. syringae pv. avellanae provides a striking example of convergent evolution of host-specificity. While both Pav lineages are part of the $P$. syringae species complex, one must go back to the origin of the species complex to find their most recent common ancestor [6]. The fact that these two lineages began causing disease on hazelnut at roughly the same time and give rise to similar disease phenotypes makes it seem unlikely that their convergent evolution occurred entirely independently. However, we find almost no evidence of genetic exchange between these lineages, and little similarity in their respective virulence gene complements.

Hazelnut decline was first described in Greece caused by phylogroup $1 \mathrm{Pav}$, yet there is strong evidence that phylogroup 2 Pav emerged first. MLSA studies show that the phylogroup $2 \mathrm{Pav}$ clade, which is restricted to Italian isolates, has over four times the genetic diversity found among the phylogroup 1 Pav strains, which include both Greek and Italian isolates [6]. This is significant since the extent of genetic diversity is usually associated with evolutionary age (baring the influence of certain evolutionary process or demographic changes). This is borne out by our molecular dating results. There is large variation in absolute divergence times depending on the substitution rate used, as rates based on fossil evidence [22] are several orders of magnitude higher than rates based on emergence of antibiotic resistant bacteria [21], diversification within hosts [21,24], or ancient DNA [25]. Despite these limitations, one clear point is that divergence times are three to ten times older for phylogroup $2 \mathrm{Pav}$ than for phylogroup $1 \mathrm{Pav}$. Indeed, even the most rapid substitution rates result in estimated divergence times for both lineages that predate the emergence of hazelnut decline by thousands of years.

The finding that Pav has been diversifying for a long period of time without being observed in the field is surprising. In Greece, Pav had a particularly heavy impact on the hazelnut cultivar Palaz during the late 1970s [3]. This cultivar was introduced from Turkey in the late 1960s where there are no records of hazelnut bacterial canker. In contrast, there has been a long history of hazelnut cultivation in Italy, although the Palaz cultivar is not grown. Italian hazelnut cultivation increased rapidly during the decades leading up to the first observed outbreak during the 1970s, going from 3500 hectares in 1945 to almost 20,000 hectares by 1990 in the province of Viterbo [26]. Much of the new cultivation in both Greece and Italy occurred on marginal lands with acidic soils, which are conditions that are likely to make hazelnut more susceptible to Pav infection.

How can the long time since Pav divergence be reconciled with the recent occurrence of hazelnut decline? Microbiological surveys of in Italy have found that wild hazelnut trees are often infected by phylogroup $2 \mathrm{Pav}$ [27], suggesting that wild trees might act as a reservoir. It is possible that phylogroup $1 \mathrm{Pav}$ are associated with wild hazelnut in Greece, but similar surveys have not been carried out. Taken together, these data strongly suggest that both Pav lineages have been cryptically infecting hazelnut trees or wild relatives for a long time, and that the emergence of hazelnut decline in the 1970s was most probably due to changes in agricultural practice.

While there is no evidence of horizontal transfer between Pav lineages, we do find a large number of genes that have been horizontally acquired from other bacteria. Over 250 ORFs from the three Pav genomes lack orthologs in any other sequenced $P$. syringae strain. This includes over 200 genes that are present in one of the phylogroup 2 Pav strains but not the other, suggesting extensive gene acquisition and loss in this lineage. Over $80 \%$ of these genes have homologs in other Proteobacteria. Many of the strain-specific genes are organized into large genomic islands with signatures of mobile elements. Two of these genomic islands are homologous to regions found in other plantassociated bacteria, although the genetic similarity is low. This suggests either that the genetic exchange occurred in the distant past or that the donor strain is only distantly related to the sequenced strains in the database. It would be interesting to sequence other hazelnut-associated bacteria such Xanthomonas arbicola pv. corylina, which is responsible for hazelnut blight and Pseudomonas fluorescens strains associated with the roots of hazelnut trees.

A remarkable feature of evolution of phylogroup 1 Pav is the extremely fluid nature of their T3SE repertoires. Like other phylogroup 1 strains, the frequency of T3SE acquisition is extremely high, with 27 T3SEs acquired since it diverged from the common ancestor of the group. However, the rate of T3SE loss is much higher than has been documented for any other $P$. syringae strain. A total of twelve Pav BP631 T3SEs are inferred to be non-functional. By comparison, the strain with the second most T3SE pseudogenes is Pto DC3000 with 
seven [16]. All of the pseudogenization events in Pav BP631 appear to have happened since it diverged from Pmp 302280 and Pan 302091. Indeed, seven of them involve T3SEs that were acquired since this divergence, meaning that they were either acquired as nonfunctional genes or that they became pseudogenes after acquisition. The frequency of T3SE gain and loss is much lower in the phylogroup $2 \mathrm{Pav}$ strains, with six and five gains for Pav Ve013 and Pav Ve037 respectively since they diverged from other phylogroup 2 strains. This is typical of the phylogroup as a whole, with three other strains that have acquired six or less T3SEs and the largest number of T3SE gains being twelve in Ppi 1704B.

Two of the Pav BP631 T3SE putative pseudogenes, avrE1 and hopM1, are notable because they are located in the CEL, which is present in all $P$. syringae strains with canonical $h r p / h r c$ type III secretion systems. AvrE1 is essential for virulence in some $P$. syringae strains [28], but is functionally redundant with HopM1 in Pto DC3000, where it suppresses salicylic acid-mediated immunity [29]. Frameshift mutations and truncations are common in hopM1, including in Pph 1448A [8], P. syringae pv. aptata DSM 50252 [4] and Pto T1 [10]. To date, all sequenced strains have had intact avrE1 genes, except for Psv 3335 [15], which has a contig break in the gene and Por 1_6, which has a premature stop codon, but has an intact hopM1 gene [14]. Homologs of $a v r E$ are also present in a number of other plant pathogens, including Erwinia amylovora and Pantoea stewartii, where it is essential for virulence [30-32]. Since $P$. syringae mutants lacking both of these T3SEs have strongly impaired virulence [33] it is unclear how Pav BP631 is able to establish infection without functional copies of either gene. It is possible that HopR1 [34] or another uncharacterized T3SE compensate for the loss of AvrE and HopM1 in hazelnut. Alternatively, a low level of translation might be initiated off the highly-atypical GTA start codon in avrE [23] or another in-frame start codon might be used, though this would be likely to have drastic effects on the $\mathrm{N}$-terminal secretion signal and there are no other obvious candidates for ribosome binding sites.

Of the twelve putatively non-functional T3SEs in Pav BP631, four have intact homologs in phylogroup 2 Pav. These include the two CEL T3SEs discussed above and two T3SEs (hopX1 and hopAI1) that were independently acquired in each Pav lineage since they diverged from their closest sequenced relatives. Furthermore, three additional T3SEs that are present in phylogroup $2 \mathrm{Pav}$ are inferred to have been lost completely in Pav BP631 since it's divergence from Pmp and Pan. This striking pattern suggests that phylogroup 1 Pav BP631 was under strong selective pressure to lose T3SEs deployed by the other Pav lineage.
The only putatively functional T3SEs that are common among the three Pav strains are HopAA1 and HopAZ1. HopAA1 is encoded in the CEL and descended from the common ancestor of $P$. syringae. It has been shown to play a role in the suppression of innate immunity in Arabidopsis [35]. Pav BP631 also carries a paralogous copy (in-paralog) of hopAA1 in addition to the one in the CEL. This paralogous hopAA1 allele is also present in the two strong Arabidopsis pathogens Pto DC3000 and Pma ES4326. One of the most interesting findings is that hopAZ1 was independently acquired in all three Pav strains, which points to HopAZ1 as a promising candidate for modulating hazelnut host specificity. Unfortunately, this T3SE has not been functionally characterized and has no conserved domains. HopAZ1 alleles are present in twelve of the 29 P. syringae strains with sequenced genomes and dispersed among four of five phylogroups. A genealogical analysis of the hop $A Z 1$ family shows strong discordance from the evolutionary history of the core genome, indicating frequent horizontal transmission of this T3SE family (Additional file 3: Figure S3).

\section{Conclusions}

Our comparative genomic analysis of three Pav isolates has further confirmed convergent evolution of two independent lineages onto hazelnut, and that this convergence is not due to genetic exchange between lineages. Furthermore, the divergence in T3SE complements suggests that the molecular mechanisms of defense evasion are distinct in each lineage. There has been particularly extensive remodeling of its T3SE repertoire in the more recently emerged lineage possibly in response to recognition by host factors that have coevolved with the T3SEs deployed by the other lineage. However, both lineages have been diversifying as hazelnut pathogens since long before the initial hazelnut decline outbreak was first documented in 1976. This suggests that changes in agricultural practice such as the propagation of new cultivars in Greece in the 1960 s and 70s and the expansion of hazelnut cultivation into marginal habitats in Italy may have provided suitable conditions for the epidemic emergence of previously cryptic pathogens. While this scenario is clearly conjecture, we now have a number of strong candidate loci to pursue. Functional characterization of these loci in the future may reveal the key steps that these two distinct lineages took in order to subvert the hazelnut immune system.

\section{Methods}

Sequencing and genome assembly followed the methods described in [36]. Briefly, cells were harvested from 
$1 \mathrm{~mL}$ of stationary-phase culture and DNA was isolated using the Gram-negative bacterial culture protocol of the Puregene Genomic DNA Purification Kit (Qiagen Canada, Toronto, ON) using double volumes of each reagent, repeating the protein precipitation step twice, and spooling the DNA during the precipitation step. Pairedend and mate-pair sequencing libraries were prepared using sample preparation kits from Illumina (San Diego, CA). DNA was sheared to 200 base pairs (bp) for the paired-end libraries and to 3 kilobases $(\mathrm{kb})$ for the matepair libraries using a Covaris S-series sample preparation system. Each library was run on a single lane of an Illumina GA IIx sequencer, for 38 cycles per end, except for the Pav Ve013 and Pav Ve037 paired-end libraries, which were run for 82 cycles per end. Paired-end reads were assembled using the CLC Genomics Workbench (Århus, Denmark), using the short-read de novo assembler for Pav BP631 and the long-read assembler for the other strains. The resultant contigs were scaffolded with the mate-pair data using SSPACE [37]. Scaffolds were ordered and oriented relative to the most closely related fully sequenced genome sequence (Pto DC3000 for PavBP631; Psy B728a for the other strains) using the contig mover tool in Mauve [20]. Automated gene prediction and annotation was carried out using the RAST annotation server [38]. These Whole Genome Shotgun projects have been deposited at DDBJ/EMBL/GenBank under the accession numbers AKBS00000000 (Pav BP631), AKCJ00000000 (Pav Ve013) and AKCK00000000 (Pav Ve037). The versions described in this paper are the first versions, AKBS01000000, AKCJ01000000 and AKCK01000000. Our methods have been shown to correctly assemble $>95 \%$ of the coding sequences, including $>98 \%$ of singlecopy genes for the fully sequenced strain $P$. syringae pv. phaseolicola (Pph) 1448A [36].

The amino acid translations of the predicted ORFs from each strain were compared to each other and to those from 26 other publically available $P$. syringae genome sequences using BLAST [39] and were grouped into orthologous gene families using orthoMCL [40]. Pav ORFs that were less than $300 \mathrm{bp}$ in length and that did not have orthologs in any other strain were excluded from further analyses. The DNA sequences of the remaining Pav-specific ORFs were compared to all other strains using BLASTn and those that matched over at least $50 \%$ of their length with an E-value $<10^{-20}$ were also excluded. The amino acid translations of the remaining $P a v$-specific genes were searched against GenBank using BLASTp to determine putative functions and the taxonomic identities of donor strains. Genomic scaffolds containing blocks of Pav-specific genes were compared to the genome sequences of the most closely related Pav reference strain and to the database strain with the most hits to ORFs in the cluster using BLASTn and similarities were visualized using the Artemis Comparison Tool [41].

Amino acid sequences of ortholog groups were aligned using MUSCLE [42], and back-translated to DNA alignments using TranslatorX [43]. PhyML [44] was used to infer phylogenies for each ortholog group and phylogenetic confidence was determined by the approximate likelihood-ratio test for branches (aLRT) method [45]. PhyML was also used to infer the core genome phylogeny by concatenating the aligned sequences of each ortholog group with one representative sequence in each strain and removing conserved alignment positions. Recombination between Pav lineages was detected by identifying gene trees in which Pav BP631 formed a monophyletic group with one or both of the other Pav strains.

In addition to the whole-genome ortholog analysis, we identified T3SE pseudogenes and gene fragments by BLASTing all of the amino acid sequences of T3SEs in the database at www.pseudomonas-syringae.org against the Pav genome sequences, as well as 24 other draft Psy genome sequences using tBLASTn. Homologous DNA sequences were extracted and examined for truncations, frameshifts, contig breaks (usually caused by the presence of transposases or other multi-copy elements disrupting the coding sequences), and chimeric proteins. Sanger sequencing was used to fill contig gaps in Pav T3SE orthologs and to confirm frameshift mutations and transposon insertions using primers flanking each gap. Sequences lacking frameshifts were translated to amino acid sequences, aligned using MUSCLE, and back-translated to DNA alignments using TranslatorX [43]. Sequences with frameshifts were added to the nucleotide alignments using MAFFT [46]. Phylogenies were inferred for each alignment using PhyML. Gains and loss of each T3SE family was mapped onto the core genome phylogeny by identifying clades in each T3SE gene tree that are congruent with the core genome phylogeny, allowing for gene loss in some lineages.

Divergence times were estimated for the most recent common ancestor of each of the Pav lineages and for $P$. syringae as a whole using the MLSA dataset from Wang et al. [6]. This included partial sequences of four protein-coding genes for ten phylogroup 1 Pav strains and twelve phylogroup 2 Pav strains, as well as 110 additional $P$. syringae strains. Analyses were carried out using an uncorrelated lognormal relaxed molecular clock in BEAST v1.6.2 [47] with unlinked trees, and substitution models, allowing for recombination between loci. The HKY substitution model was used with gammadistributed rate variation, with separate partitions for codon positions $1+2$ and for third positions. Substitution rates were set to published rates based on the split of Escherichia coli and Salmonella [22] and the 
emergence of methicillin resistant Staphylococcus aureus (MRSA) [21]. Two independent Markov chains were run for 50 Million generations and results were combined for parameter estimates.

\section{Additional files}

\section{Additional file 1: Figure S1. BLASTn-based comparison of Pav Ve013, Psy B728a and Xanthomonas campestris 8004 showing a 110 kb insertion in Pav Ve013 with portions that are homologous to three different regions in the $X$. campestris 8004 genome. \\ Additional file 2: Figure S2. BLASTn-based comparison of Pav Ve013, Pav Ve037, Psy B728a and Pseudomonas fluorescens SBW25 showing large insertions in both Pav strains which lack homology to each other except for a central core homologous to an integrative conjugative element (ICE) in P. fluorescens SBW25.}

Additional file 3: Figure S3. Gene tree for hopAZ homologs from all sequenced $P$. syringae strains. Pav sequences, which are colored in red, are found in three major subclades. Numbers above branches indicate aLRT branch support values.

\section{Abbreviations}

CEL: Conserved effector locus; EEL: Exchangeable effector locus; MLSA: Multilocus sequence analysis; MYA: Millions of years ago; T3SE: Type III secreted effector; Bp: Base pairs; Kb: Kilobases; Pan: P. syringae pv. actinidiae; Pav: Pseudomonas syringae pv. avellanae; Pja: P. syringae pv. japonica; Pmp: P. syringae pv. morsprunorum; Ppi: P. syringae pv. pisi; Psy: P. syringae pv. syringae; Pto: P. syringae pv. tomato; Ptt: P. syringae pv. aptata.

\section{Competing interest}

The authors declare no competing interests.

\section{Acknowledgements}

This work was supported by grants from the Natural Sciences \& Engineering Research Council (NSERC) of Canada and the Canada Research Chairs Program to DSG.

\section{Author details}

'Department of Cell and Systems Biology, University of Toronto, 25 Willcocks St., Toronto, ON M5S 3B2, Canada. ${ }^{2}$ Center for the Analysis of Genome Evolution \& Function, University of Toronto, 25 Willcocks St., Toronto, ON M5S 3B2, Canada. ${ }^{3}$ C.R.A.- Fruit Crops Research Centre, Via di Fioranello, 52; I00134 Rome, Italy.

\section{Authors' contributions}

DSG, HEOB and MS conceived and designed the experiments. CY, PF, LY, JZ and PWW performed the experiments. HEOB, ST and YG analyzed the data. DSG Contributed reagents and materials. DSG, HEOB and MS wrote the paper. All authors read and approved the final manuscript.

Received: 6 January 2012 Accepted: 16 July 2012

Published: 16 July 2012

\section{References}

1. Hwang MSH, Morgan RL, Sarkar SF, Wang PW, Guttman DS: Phylogenetic characterization of virulence and resistance phenotypes of Pseudomonas syringae. Appl Environ Microbiol 2005, 71:5182-5191.

2. Sarkar SF, Guttman DS: Evolution of the core genome of Pseudomonas syringae, a highly clonal, endemic plant pathogen. Appl Environ Microbiol 2004, 70:1999-2012.

3. Scortichini M: Bacterial canker and decline of European hazelnut. Plant Dis 2002, 86:704-709.

4. Baltrus DA, Nishimura MT, Romanchuk A, Chang JH, Mukhtar MS, Cherkis K, Roach J, Grant SR, Jones CD, Dangl JL: Dynamic evolution of pathogenicity revealed by sequencing and comparative genomics of 19 Pseudomonas syringae isolates. PLoS Pathog 2011, 7:e1002132.

5. Marcelletti S, Ferrante P, Petriccione M, Firrao G, Scortichini M: Pseudomonas syringae pv. actinidiae draft genomes comparison reveal strain-specific features involved in adaptation and virulence to Actinidia species. PLoS One 2011, 6:e27297.

6. Wang PW, Morgan RL, Scortichini M, Guttman DS: Convergent evolution of phytopathogenic pseudomonads onto hazelnut. Microbiology 2007, 153:2067-2073.

7. Cai R, Lewis J, Yan S, Liu H, Clarke CR, Campanile F, Almeida NF, Studholme DJ, Lindeberg M, Schneider D, et al: The plant pathogen Pseudomonas syringae pv. tomato is genetically monomorphic and under strong selection to evade tomato immunity. PLoS Pathog 2011, 7:e1002130.

8. Joardar V, Lindeberg M, Jackson RW, Selengut J, Dodson R, Brinkac LM, Daugherty SC, DeBoy R, Durkin AS, Giglio MG, et al: Whole-genome sequence analysis of Pseudomonas syringae pv. phaseolicola 1448A reveals divergence among pathovars in genes involved in virulence and transposition. J Bacterio/ 2005, 187:6488-6498.

9. Studholme DJ, Gimenez Ibanez S, MacLean D, Dangl JL, Chang JH, Rathjen JP: A draft genome sequence and functional screen reveals the repertoire of type III secreted proteins of Pseudonomas syringae pathovar tabaci 11528. BMC Genomics 2009, 10:395.

10. Almeida NF, Yan S, Lindeberg M, Studholme DJ, Schneider DJ, Condon B, Liu H, Viana CJ, Warren A, Evans C, et al: A draft genome sequence of Pseudomonas syringae pv. tomato $\mathrm{T} 1$ reveals a type III effector repertoire significantly divergent from that of Pseudomonas syringae pv. tomato DC3000. Mol Plant Microbe Interact 2009, 22:52-62.

11. Farrer RA, Kemen E, Jones JDG, Studholme DJ: De novo assembly of the Pseudomonas syringae pv. syringae B728a genome using Illumina/Solexa short sequence reads. FEMS Microbiol Lett 2009, 291:103-111.

12. Green S, Studholme DJ, Laue BE, Dorati F, Lovell H, Arnold D, Cottrell JE, Bridgett S, Blaxter M, Huitema E, et al: Comparative genome analysis provides insights into the evolution and adaptation of Pseudomonas syringae pv. aesculi on Aesculus hippocastanum. PLoS One 2010, 5:e10224

13. Qi M, Wang D, Bradley CA, Zhao Y: Genome sequence analyses of Pseudomonas savastanoi pv. glycinea and subtractive hybridization-based comparative genomics with nine pseudomonads. PLoS One 2011, 6:e16451.

14. Reinhardt JA, Baltrus DA, Nishimura MT, Jeck WR, Jones CD, Dangl JL: De novo assembly using low-coverage short read sequence data from the rice pathogen Pseudomonas syringae pv. oryzae. Genome Res 2009, 19:294-305

15. Rodríguez-Palenzuela P, Matas IM, Murillo J, López-Solanilla E, Bardaji L, Pérez-Martínez I, Rodríguez-Moskera ME, Penyalver R, López MM, Quesada $J \mathrm{M}$, et al: Annotation and overview of the Pseudomonas savastanoi pv. savastanoi NCPPB 3335 draft genome reveals the virulence gene complement of a tumour-inducing pathogen of woody hosts. Environ Microbiol 2010, 12:1604-1620.

16. Buell CR, Joardar V, Lindeberg M, Selengut J, Paulsen IT, Gwinn ML, Dodson RJ, Deboy RT, Durkin AS, Kolonay JF, et al: The complete genome sequence of the Arabidopsis and tomato pathogen Pseudomonas syringae pv. tomato DC3000. Proc Natl Acad Sci U S A 2003, 100:10181-10186.

17. Feil H, Feil WS, Chain P, Larimer F, DiBartolo G, Copeland A, Lykidis A, Trong S, Nolan M, Goltsman E, et al: Comparison of the complete genome sequences of Pseudomonas syringae pv. syringae B728a and pv. tomato DC3000. Proc Natl Acad Sci U S A 2005, 102:11064-11069.

18. Fox J, Weisberg S: An R Companion to Applied Regression. 2nd edition. Thousand Oaks CA: Sage Publications; 2011.

19. R Deveolpment Core Team: R: A Language and Environment for Statistical Computing. Vienna, Austria: R Foundation for Statistical Computing; 2011.

20. Darling AE, Mau B, Perna NT: progressiveMauve: multiple genome alignment with gene gain, loss and rearrangement. PLoS One 2010, 5:e11147.

21. Nübel U, Dordel J, Kurt K, Strommenger B, Westh H, Shukla SK, Žemličková $H$, Leblois $R$, Wirth $T$, Jombart $T$, et al: A timescale for evolution, population expansion, and spatial spread of an emerging clone of methicillin-resistant Staphylococcus aureus. PLoS Pathog 2010, 6:e1000855.

22. Ochman H, Wilson AC: Evolution in bacteria: evidence for a universal substitution rate in cellular genomes. J Mol Evol 1987, 26:74-86.

23. Kim SW, Jung WH, Ryu JM, Kim JB, Jang HW, Jo YB, Jung JK, Kim JH: Identification of an alternative translation initiation site for the Pantoea ananatis lycopene cyclase (crth) gene in $E$. coli and its evolutionary conservation. Protein Expr Purif 2008, 58:23-31.

24. Morelli G, Didelot X, Kusecek B, Schwarz S, Bahlawane C, Falush D, Suerbaum S, Achtman M: Microevolution of Helicobacter pylori during prolonged infection of single hosts and within families. PLOS Genet 2010 6:e1001036. 
25. Bos KI, Schuenemann VJ, Golding GB, Burbano HA, Waglechner N, Coombes BK, Mcphee JB, Dewitte SN, Meyer M, Schmedes S, et al: A draft genome of Yersinia pestis from victims of the Black Death. Nature 2011, 478:506-510.

26. Scortichini M: The problem caused by Pseudomonas avellanae on hazelnut in Italy. Proceedings of the Fifth International Congress on Hazelnut. Acta Horticulturae 2001, 556:503-508.

27. Scortichini M, Marchesi U, Angelucci L: Occurrence of Pseudomonas avellanae (Psallidas) Janse et al. and related pseudomonads on wild Corylus avellana trees and genetic relationships with strains isolated from cultivated hazelnuts. J Phytopathol 2000, 148:523-532.

28. Lorang JM, Keen NT: Characterization of avrE from Pseudomonas syringae pv. tomato: a hrp-linked avirulence locus consisting of at least two transcriptional units. MPMI 1995, 8:49-57.

29. DebRoy S, Thilmony R, Kwack Y-B, Nomura K, He SY: A family of conserved bacterial effectors inhibits salicylic acid-mediated basal immunity and promotes disease necrosis in plants. Proc Natl Acad Sci USA 2004, 101:9927-9932

30. Bogdanove AJ, Kim JF, Wei Z, Kolchinsky P, Charkowski AO, Conlin AK, Collmer A, Beer SV: Homology and functional similarity of an hrp-linked pathogenicity locus, $d s p E F$, of Erwinia amylovora and the avirulence locus avrE of Pseudomonas syringae pathovar tomato. Proc Natl Acad Sci USA 1998, 95:1325-1330.

31. Frederick RD, Ahmad M, Majerczak DR, Arroyo-Rodríguez AS, Manulis S, Coplin DL: Genetic organization of the Pantoea stewartii subsp. stewartii hrp gene cluster and sequence analysis of the $h r p A, h r p C, h r p N$, and wtsE operons. MPMI 2001, 14:1213-1222.

32. Gaudriault S, Malandrin L, Paulin JP, Barny MA: DspA, an essential pathogenicity factor of Erwinia amylovora showing homology with AvrE of Pseudomonas syringae, is secreted via the Hrp secretion pathway in a DspB-dependent way. Mol Microbiol 1997, 26:1057-1069.

33. Badel JL, Shimizu R, Oh H-S, Collmer A: A Pseudomonas syringae pv. tomato avrE1/hopM1 mutant is severely reduced in growth and lesion formation in tomato. Mol Plant Microbe Interact 2006, 19:99-111.

34. Kvitko BH, Park DH, Velasquez AC, Wei CF, Russell AB, Martin GB, Schneider DJ, Collmer A: Deletions in the repertoire of Pseudomonas syringae pv. tomato DC3000 type III secretion effector genes reveal functional overlap among effectors. PLOS Pathog 2009, 5:e1000388.

35. Li X, Lin H, Zhang W, Zou Y, Zhang J, Tang X, Zhou J-M: Flagellin induces innate immunity in nonhost interactions that is suppressed by Pseudomonas syringae effectors. Proc Natl Acad Sci USA 2005, 102:12990-12995.

36. O'Brien HE, Gong Y, Fung P, Wang PW, Guttman DS: Use of low-coverage, large-insert, short-read data for rapid and accurate generation of enhanced-quality draft Pseudomonas genome sequences. PLoS One 2011, 6:e27199.

37. Boetzer M, Henkel CV, Jansen HJ, Butler D, Pirovano W: Scaffolding preassembled contigs using SSPACE. Bioinformatics (Oxford, England) 2011 27:578-579.

38. Aziz RK, Bartels D, Best AA, DeJongh M, Disz T, Edwards RA, Formsma K, Gerdes S, Glass EM, Kubal M, et al: The RAST server: rapid annotations using subsystems technology. BMC Bioinforma 2008, 9:75.

39. Altschul SF, Madden TL, Schäffer AA, Zhang J, Zhang Z, Miller W, Lipman DJ: Gapped BLAST and PSI-BLAST: a new generation of protein database search programs. Nucleic Acids Res 1997, 25:3389-3402.

40. Li L, Stoeckert CJ, Roos DS: OrthoMCL: identification of ortholog groups for eukaryotic genomes. Genome Res 2003, 13:2178-2189.

41. Carver TJ, Rutherford KM, Berriman M, Rajandream M-A, Barrell BG, Parkhill J: ACT: the Artemis Comparison Tool. Bioinformatics (Oxford, England) 2005, 21:3422-3423

42. Edgar RC: MUSCLE: multiple sequence alignment with high accuracy and high throughput. Nucleic Acids Res 2004, 32:1792-1797.

43. Abascal F, Zardoya R, Telford MJ: TranslatorX: multiple alignment of nucleotide sequences guided by amino acid translations. Nucleic Acids Res 2010, 38:W7-W13.

44. Guindon S, Gascuel O: A simple, fast, and accurate algorithm to estimate large phylogenies by maximum likelihood. Syst Biol 2003, 52:696-704.

45. Anisimova M, Gascuel O: Approximate likelihood-ratio test for branches: A fast, accurate, and powerful alternative. Syst Biol 2006, 55:539-552.

46. Katoh K, Misawa K, Kuma K-, Miyata T: MAFFT: a novel method for rapid multiple sequence alignment based on fast Fourier transform. Nucleic Acids Res 2002, 30:3059-3066.
47. Drummond AJ, Rambaut A: BEAST: Bayesian evolutionary analysis by sampling trees. BMC Evol Biol 2007, 7:214.

doi:10.1186/1471-2180-12-141

Cite this article as: $O^{\prime}$ Brien et al: Extensive remodeling of the Pseudomonas syringae pv. avellanae type III secretome associated with two independent host shifts onto hazelnut. BMC Microbiology 2012 $12: 141$

\section{Submit your next manuscript to BioMed Central and take full advantage of:}

- Convenient online submission

- Thorough peer review

- No space constraints or color figure charges

- Immediate publication on acceptance

- Inclusion in PubMed, CAS, Scopus and Google Scholar

- Research which is freely available for redistribution

Submit your manuscript at www.biomedcentral.com/submit
C) Biomed Central 